\title{
Extensible Portfolio of Forecasting Methods for ERP Systems: An Integration Approach
}

\author{
Jānis Pekša \\ Riga Technical University, Riga, Latvia
}

\begin{abstract}
Enterprise resource planning (ERP) systems are large, modular enterprise applications designed for most of the company's business processes. They include a range of different forecasting methods. The paper analyses the existing forecasting methods in ERP systems and provides a comparison of forecasting methods in ERP systems. It considers the problem of prediction integration in ERP systems and describes the general process by a conceptual model based on academic literature from forecasting with ERP systems. The study provides an integration approach, which is the most suitable one for providing forecasting functions in ERP systems.
\end{abstract}

Keywords - ERP systems, forecasting, forecasting methods.

\section{INTRODUCTION}

ERP systems are large modular enterprise applications intended for execution of most of enterprise business processes. They are primarily geared towards transaction processing. However, many modules contain complex forecasting methods and are part of decision-making logic [1]. Forecasting is a process for predicting the future based on past data, and most often through a trend analysis [2]. Prediction is one of the cases that business needs in high demand to gain higher profit and continue successfully business processes [3]. ERP systems have limited forecasting capabilities that are implemented into a hard-coded code [4]. Enterprises spend much money to modify existing methods to satisfy their requirements [5]. Some of ERP systems do not have enough forecasting functionality.

The present paper analyses existing forecasting methods for ERP systems gathering an extensible portfolio. It considers a forecasting integration problem in ERP systems, where software as a service (SaaS) comes in place giving a centrally hosted solution. The study represents an overall process with the help of conceptual model driven by academic literature found on forecasting along with ERP systems. The author examines existing forecasting methods in ERP systems and represents an integration solution design and approach to forecast implementation using SaaS.

The rest of the paper is organised as follows: Section II discusses related work, Section III proposes an integration solution, and Section IV provides summary and conclusions.

\section{RELATED WORK}

Evidence of forecasting methods used in ERP systems is gathered in this section also, compiling integration solutions that exist in ERP systems.

\section{A. Forecasting Methods in ERP Systems}

As already known, there are three major categories of forecasts: economic forecasts [6], technological forecasts [7] and demand forecasts provided in [8] representing forecast evolution. There are many types of forecasting methods in these three categories. The present paper considers only quantitative forecasting methods, although there are also qualitative forecasting methods such as:

- Executive Opinion - uses a composite forecast prepared by several individual experts [9];

- Market Survey - a forecast method that involves speaking to members of the target audience [10];

- Delphi Method - is used in policymaking and organisational decision making [11];

- Salesforce - is used to view the best estimate of revenue generated in a specified time frame [12].

However, quantitative forecasting methods are based on mathematical models, and they are objective [13]. These methods can only be applied when both conditions are contented. Under the first condition, numerical information is available about the past and under the second condition it can be assumed that some of the aspects of previous patterns will continue in the future [14]. There are many quantitative forecasting methods. To reduce the number of methods to be considered, the time-series forecasting is selected. Overall representation of forecasts, forecasting methods, and forecasting models is shown in Fig. 1.

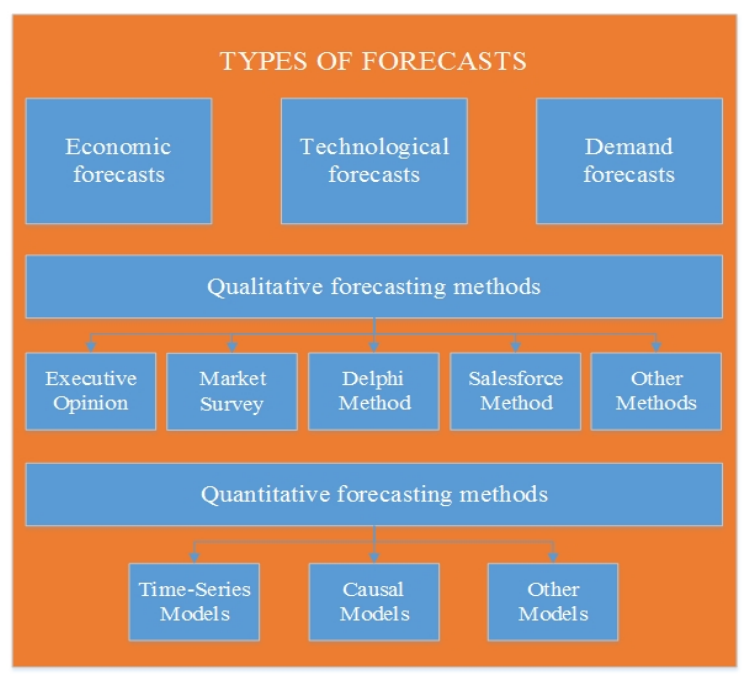

Fig. 1. Representation of forecasts and forecasting methods. 
Time-series forecasting uses historical data as the basis for estimating future outcomes. There are two types of time series - continuous and discrete. For discrete time series, it is the time axis that is discrete while in continuous time series the observed variable is typically a continuous variable recorded continuously on a trace. Several forecasting procedures are based on a time-series model [15].

To readily perceive the nature of each model, they are explained. Further, one of the largest ERP systems is considered, identified in the SAP ERP system and Microsoft Dynamics AX:

- Autoregressive integrated moving average - also known as ARIMA, is fitted to time series data to understand the data better or to predict future points in the series [16];

- Autoregressive tree model - historically known as ARTxp algorithm from Microsoft SQL Server 2005 supporting cross-validation. However, an ART model is a cloggy linear autoregressive model, in which the borders are defined by a decision tree, and the leaves of the decision tree contain linear autoregressive models [17];

- Automatic model selection - the system analyses the historical data and then selects the most eligible model [18];

- Box-Jenkins method - to find the best fit of a time-series model to past values of a time series [19] it applies autoregressive moving average (ARMA) and uses ARIMA as a set;

- Linear regression - a statistical method that allows summarising and studying relationships between two continuous quantitative variables [20] and it is the bestknown demand forecasting method for a trendy demand [21];

- Moving average - an analysis tool that smoothes out price data by creating a constantly updated average price [22];

- Holt-Winters method - one of the time series with the trend but with seasonality [23] used to forecast for "what-if" analysis [24], and an additive method is one of popular methods [25];

- Weighted moving average - puts more weight on recent data and less on past data [26].

Collection of time-series models allow dividing each ERP system, which has these models enabled, using a design evaluation method like an observational one in a field study to find the use of forecasting methods in multiple ERP systems [27]. For example, SAP ERP and Microsoft Dynamics AX systems are taken to compare each other. These two ERP systems, which are one of the largest and most significant systems, are compared taking into account three criteria: "+" has such a method, "-" does not have this method and " $+/-$ " has this method partially. All the criteria are summarised in a single table (see Table I).
TABLE I

COMPARISON OF FORECASTING METHODS IN ERP SYSTEMS

\begin{tabular}{|l|c|c|}
\hline \multicolumn{1}{|c|}{ Time-series models/methods } & & \\
& & \\
& & \\
& & \\
\hline Autoregressive integrated moving average & + & + \\
\hline Autoregressive tree model & - & + \\
\hline Automatic model selection & + & - \\
\hline Box-Jenkins method & + & $+/-$ \\
\hline Holt-Winters method & + & + \\
\hline Moving average & + & + \\
\hline Linear regression & + & - \\
\hline Weighted moving average & + & + \\
\hline
\end{tabular}

ERP systems mostly have the same models and methods, but some are unique. However, in SAP ERP system there is not an auto-regressive tree model and in Microsoft Dynamics AX there are not automatic model selection, some Box-Jenkins method and linear regression. It is worth mentioning that Microsoft Dynamics AX can provide a configuration environment where users can make from scratch linear regression. SAP ERP system shows the advantage of forecasting models and methods count that are available by default in the German enterprise resource planning system.

\section{B. Forecasting Process in ERP Systems}

To better understand the current situation of the forecasting process in ERP systems, forecasting functionality of Microsoft Dynamics AX is analysed in detail and some observations are made about forecasting in SAP ERP systems.

Microsoft Dynamics AX provides a section for demand forecast generation with AX 2012 R3 version. Basically, historical transactional data from AX transactional database are gathered and a staging table is created. The staging data are afterwards moved to an analysis services mining model of AX. With small customization, users can plug different data sources into the selected table, such as Excel files and comma-separated files. It allows users to create a demand forecast that takes historical data distributed across multiple systems. The mining model uses prediction methods for time series analysis services to calculate the initial forecasts. The parameters for these forecasting methods are managed in AX 2012 R3. Then, the forecast and historical data, as well as the changes made in the demand forecast for previous iterations, are made available in the SQL Server data cube. Microsoft Excel is used to connect to the demand forecasting cube so that users can visualise and modify initial forecasts. The manual adjustments are to be selected back to the cube before they can import the AX 2012 R3. Since there is only one demand forecasting cube at any given time, there is only one forecast. Finally, starting with the 2012 AX, adjusted forecasts of predicted models can be imported back to the AX 2012 R3 database. The overall flow of the forecasting process in AX is shown in Fig. 2. 


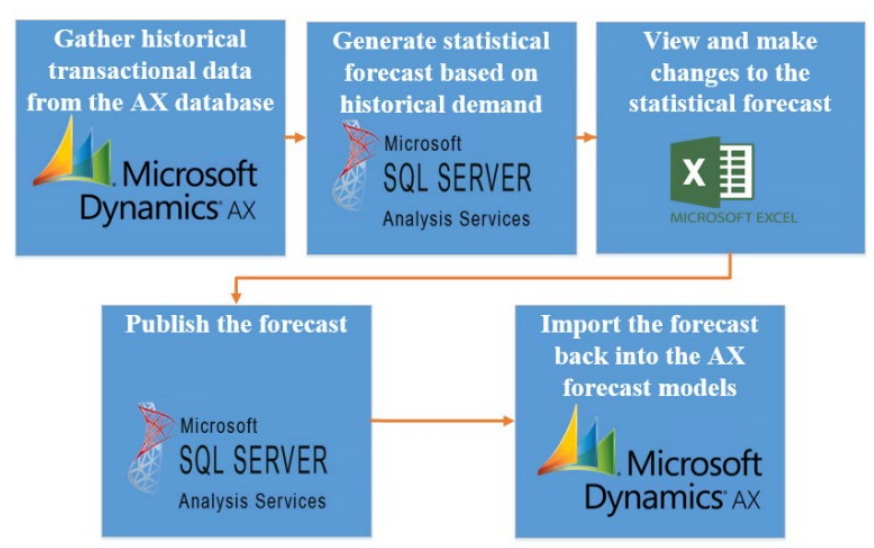

Fig. 2. Forecast process in Microsoft Dynamics AX 2012 R3.

\section{Integration Approaches in ERP Systems}

To identify the most common integration approach for forecasting methods that can be used in ERP systems, the best way is to gather integration approaches from literature and select one of integration approaches that is most appropriate for this trend. The following integration approaches are considered: internal integration, service-oriented, and SaaS.

In the case of internal integration, forecast methods are implemented and executed in ERP core systems. Research [28] analyses how procurement ambiguities of renewable resources can be considered in the basic data structures of ERP systems, namely bill of materials (BOM), work schedules and time schedules. Accordingly, the authors describe certain variation methods as general possibilities to handle uncertainty. This analysis makes it possible to understand how the existing system can be improved so that it is more efficient and not so much saturated with business failures. Adding system kernel forecasting to the ERP system can be useful for future optimization and error reduction.

Service-oriented approaches as indicated in [29] assume bottom-up thinking where the ERP system is segmented in provisions of business processes or business scenario models; various technical complexions are quantified to help solve the ERP customization estimation problem. They focus on the analytical level of a business process or a scenario model and proceed with delivering estimation figures at that level.

SaaS is typically accessed by users using a thin client via a web browser. SaaS already remains a benchmark for adding ERP system models and approaches from outside in recent years [30]. A lot of literature provides SaaS techniques and approaches in ERP systems. In [31], novel architecture for the field of industrial automation is described, the goals of which are: 1) computation of optimal production plans; 2) automated usage of the optimised plans; 3) flexibility and reusability during development and maintenance; and 4) seamless transition from current practice to the approach introduced herein. Research [30] review SMEs cloud computing in general, and SaaS are popular solutions to share the costs for IT service development and activities. Accordingly, the task of data analytics for product sales forecasting is a hopeful application for a new cloud service model. Those positive aspects and opportunities for SaaS lead to use this approach for integration of forecast methods into ERP systems. Next section provides a detailed integration solution, which can lead IT companies to integrate typical models into ERP systems.

\section{INTEGRATION SOLUTION}

The solution to integration is the so-called ERP system extended approach that focuses on interfacing between external components and ERP system and integration protocols ought to enable for plugging-in appropriate components when necessary. Some data cannot be stored in the ERP system because they can cause an overload to the system. SaaS comes in place serving as a mechanism to automate certain decisions to be made. The integration of external solution source allows not adversely affecting ERP system resources. Good forecasts require much data to work with predictions. ERP systems are not designed to overload the system with excessive temporary data. From a business aspect, the overall business model is represented by separation from the ERP system and SaaS in Fig. 3 using a business process model and notation (BPMN) with a forecasting SaaS approach.

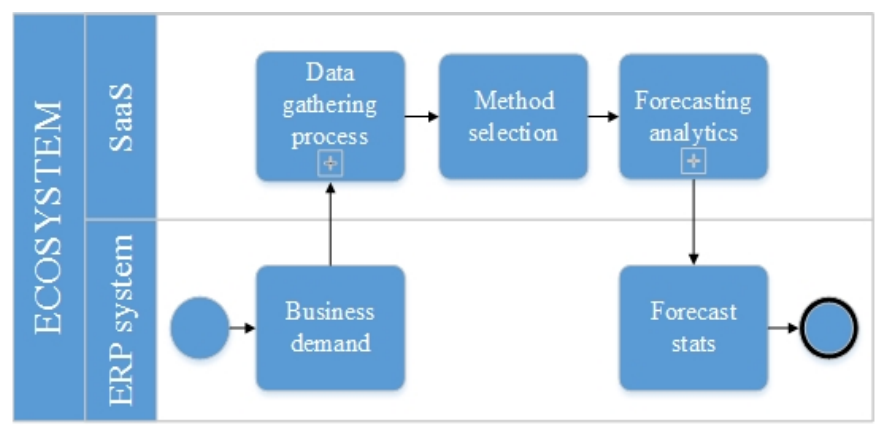

Fig. 3. BPMN process with a forecasting SaaS approach.

Ecosystem uses the connection between SaaS and ERP system to perform analytics eliminating heavy load on ERP system resources while allowing one to gather analytics of forecast stats returning a simple result into ERP system. Basically, SaaS gets the command from the ERP system triggered from time to time or by a super user of ERP systems. After the trigger is active, a data gathering process starts proceeding with method selection for the best scenario. With minimum latency, the ERP system gets forecast stats, and automatically system can act according to the specific demand.

More specifically, links between each process conceptual model design are represented in Fig. 4 that shows an example of linear regression forecast methods cycle in SaaS. There are also external data that are taken from external sources, such as public datasets, and more external sources are added to increase the accuracy of the result. 


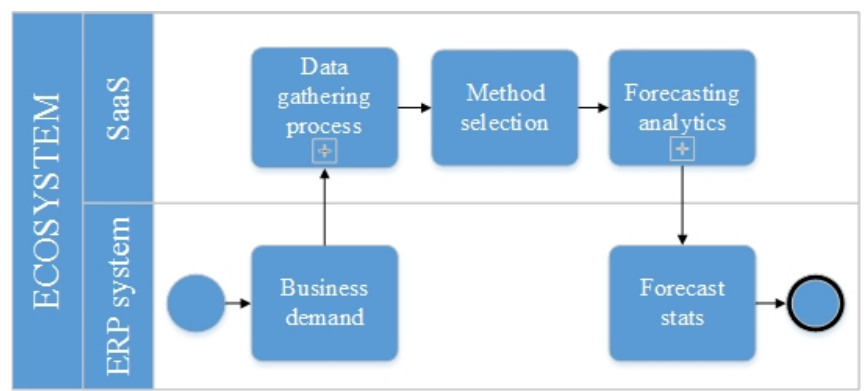

Fig. 4. A conceptual model of SaaS approach.

ERP systems can use multiple SaaS for those business process needs. The SaaS approach conceptual model uses forecasting methods to work with the automatically selected correct forecasting method. External data can be added for better results, but also it is possible to use ERP system data that are available through existing business processes. Better results in future are possible to have by adding more forecasting methods, such as machine learning for any model used in SaaS forecasting framework, for example, neural networks (NN).

Figure 5 represents a sequence diagram where the demand for each signal and the subsequent sequence to the result are reflected. Unified modelling language sequence diagram is used for a better representation of the forecasting process. For forecasting, a powerful framework such as Apache Sparks will be used in future. Apache Sparks is an open-source clustercomputing framework, where it is possible to work with big data, and it allows using external libraries like MLlib (Apache Sparks Machine Learning (ML) library). Prediction with MLlib can use forecasting models such as ARIMA, and it is possible to code new models that do not exist in MLlib as default models.

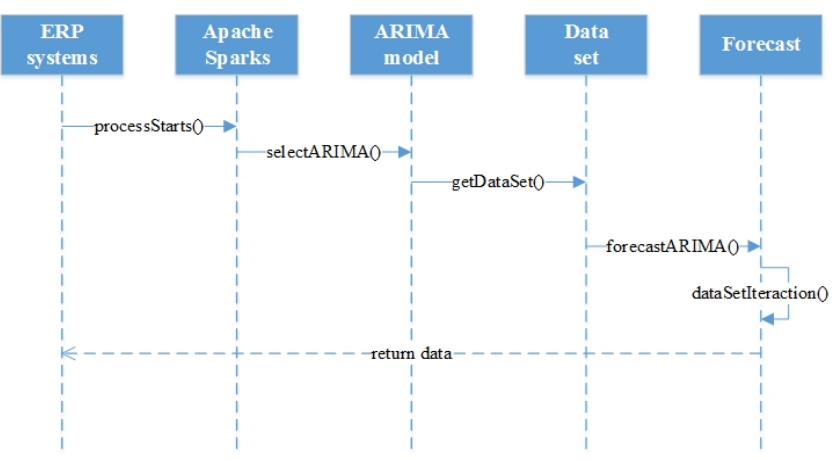

Fig. 5. Sequence diagram of the forecasting process.

Apache Sparks allows the ARIMA model to input external data sets into the SaaS approach. In the future, this open-source cluster-computing framework can be used to carry out a real experiment with big data.

\section{CONCLUSION}

Forecasting nowadays is very important for business processes that are found in ERP systems. Those business processes can gain many investment savings for companies and add extra value to business processes as it is possible to predict future indicators. However, the forecast is actually more than a decade old method, it increases the accuracy of mathematical calculations, which allows increasing prediction accuracy.

Results that have been achieved indicate that the integration approach with Apache Sparks open-source cluster-computing framework can be used for big data problem with the SaaS approach.

The summary and findings which have already been reported in this article are a starting point for further results. Next steps are to add machine learning to any model used in the SaaS forecasting framework problem. NN can adapt to the variable input data so that the network can create the best possible result without the need to modify the output criteria. Input data are restored every time data are received, they are entered into the $\mathrm{NN}$ and the accuracy of the estimate increases. The main goal is to connect this process with live stream data and add knowledgeable resources. Those live streams would help increase the accuracy of the forecast.

\section{REFERENCES}

[1] C. W. Holsapple, M. P. Sena, "ERP plans and decision-support benefits," in Decision Support Systems, vol. 38, no. 4, pp. 575-590, 2005. https://doi.org/10.1016/i.dss.2003.07.001

[2] S. Makridakis, S. C. Wheelwright, and R. J. Hyndman, Forecasting methods and applications, 3rd edn., New York, USA: John Wiley \& Sons, 1998.

[3] A. I. Dimitras, S. H. Zanakis, and C. Zopounidis, "A survey of business failures with an emphasis on prediction methods and industrial applications," European Journal of Operational Research, vol. 90, no. 3, pp. 487-513, 1996. https://doi.org/10.1016/0377-2217(95)00070-4

[4] F. R. Jacobs, and R. B. Chase, Operations and supply chain management. McGraw-Hill Education, 14 ed., 2013.

[5] B. Aslan, M. Stevenson, and L. C. Hendry, "Enterprise Resource Planning systems: An assessment of applicability to Make-To-Order companies," Computers in Industry, vol. 63, No. 7, pp. 692-705, 2012. https://doi.org/10.1016/j.compind.2012.05.003

[6] J. P. Martino, Technological forecasting for decision making. New York, USA: McGraw-Hill, Inc., 1993.

[7] R. S. Pindyck, and D. L. Rubinfeld, Econometric models and economic forecasts, $4^{\text {th }}$ ed., Boston, Mass, USA: Irwin/McGraw-Hill, 1998.

[8] R. Güllü, "On the value of information in dynamic production/inventory problems under forecast evolution," Naval Research Logistics (NRL), vol. 43, no. 2, pp. 289-303, 1996. https://doi.org/10.1002/(SICI)15206750(199603)43:2<289::AID-NAV8>3.0.CO;2-6

[9] C. Browne, A. Di Battista, T. Geiger, and T. Gutknecht, "The executive opinion survey: The voice of the business community," The Global Competitiveness Report 2014-2015, pp. 69-78.

[10] C. A. Moser, "Survey Methods in Social Investigation," Mar. 2017. https://doi.org/10.4324/9781315241999

[11] S. R. Brady, "Utilizing and adapting the Delphi method for use in qualitative research," International Journal of Qualitative Methods, vol. 14, no. 5, 2015. https://doi.org/10.1177/1609406915621381

[12] B. Balboni, and H. Terho, "Outward-looking and future-oriented customer value potential management: The sales force value appropriation role," Industrial Marketing Management, vol. 53, pp. 181-193, 2016. https://doi.org/10.1016/j.indmarman.2015.05.022

[13] W. W. Wei, "Time series analysis," in the Oxford Handbook of Quantitative Methods in Psychology: vol. 2. 2006.

[14] R. J. Hyndman, and G. Athanasopoulos, Forecasting: principles and practice, OTexts, 2013.

[15] C. Chatfield, "Time-series forecasting," New York, CRC Press, 2000. https://doi.org/10.1201/9781420036206

[16] A. J. Conejo, M. A. Plazas, R. Espinola, and A. B. Molina, "Day-ahead electricity price forecasting using the wavelet transform and ARIMA models," IEEE transactions on power systems, vol. 20, no. 2, pp. 1035-1042, 2005. https://doi.org/10.1109/TPWRS.2005.846054 
[17] C. Meek, D. M. Chickering, and D. Heckerman, "Autoregressive Tree Models for Time-Series Analysis," Proceedings of the 2002 SIAM International Conference on Data Mining, pp. 229-244, Apr. 2002. https://doi.org/10.1137/1.9781611972726.14

[18] N. Kourentzes, "On intermittent demand model optimisation and selection," International Journal of Production Economics," vol. 156, pp. 180-190, Oct. 2014. https://doi.org/10.1016/j.ijpe.2014.06.007

[19] M. Hibon, and S. Makridakis, "ARMA models and the Box-Jenkins methodology," Journal of Forecasting, vol. 16, no. 3, 1997. https://doi.org/10.1002/(SICI)1099-131X(199705)16:3<147::AIDFOR652>3.0.CO;2-X

[20] S. Kraft, S. Pacheco-Sanchez, G. Casale, and S. Dawson, "Estimating Service Resource Consumption from Response Time Measurements," Proceedings of the 4th International ICST Conference on Performance Evaluation Methodologies and Tools, 2009 https://doi.org/10.4108/ICST.VALUETOOLS2009.7526

[21] M. E. Nenni, L. Giustiniano, and L. Pirolo, "Demand Forecasting in the Fashion Industry: A Review," International Journal of Engineering Business Management, vol. 5, p. 37, Jan. 2013 https://doi.org/10.5772/56840

[22] S. Belbag, M. Çimen, S. Tarim, and A. Tas, "A Research on Corporate Enterprise Resource Planning (ERP) Systems used for Supermarke Supply Chain Inventory Management in Turkey," European Journal of Scientific Research, vol. 38, no. 3, pp. 486-499, 2009.

[23] F. Gulyassy, and M. Hoppe, Materials planning with SAP, SAP Press, 2009.

[24] L. Houghton, and D. V. Kerr, "A study into the creation of feral information systems as a response to an ERP implementation within the supply chain of a large government-owned corporation," International Journal of Internet and Enterprise Management, vol. 4, no. 2, p.135, 2006. https://doi.org/10.1504/IJIEM.2006.010239

[25] V. C. Sugiarto, R. Sarno, and D. Sunaryono, "Sales forecasting using Holt-Winters in Enterprise Resource Planning at sales and distribution module," in International Conference on Information \& Communication
Technology
and
Systems
(ICTS),
2016.

[26] J. W. Taylor, "Multi-item sales forecasting with total and split exponential smoothing," Journal of the Operational Research Society, vol. 62, no. 3, pp. 555-563, Mar. 2011. https://doi.org/10.1057/jors.2010.95

[27] A. R. Hevner, S. T. March, J. Park, and S. Ram, "Design science in information systems research," MIS Quarterly, vol. 28, no. 1, pp.75-105, 2004. https://doi.org/10.2307/25148625

[28] S. Friedemann, and M. Schumann, "How to consider supply uncertainty of renewable resources in the basic data structures of ERP-systems," in Innovation and Future of Enterprise Information Systems, F. Piazolo, and M. Felderer, Eds., Berlin, Heidelberg: Springer, 2013, pp. 253-269. https://doi.org/10.1007/978-3-642-37021-2 19

[29] S. Parthasarathy and M. Daneva, "An approach to estimation of degree of customization for ERP projects using prioritized requirements," Journal of Systems and Software, vol. 117, pp. 471-487, Jul. 2016. https://doi.org/10.1016/j.jss.2016.04.006

[30] F. Aulkemeier, R. Daukuls, M. E. Iacob, J. Boter, J. Van Hillegersberg, and S. De Leeuw, "Sales Forecasting as a Service-A Cloud-based Pluggable E-Commerce Data Analytics Service," Proc. 18th International Conference on Enterprise Information Systems, vol. 2, ICEIS, Rome, 2016, pp. 345-352. http://doi.org/10.5220/0005915903450352

[31] A. Girbea, C. Suciu, S. Nechifor, and F. Sisak, "Design and implementation of a service-oriented architecture for the optimization of industrial applications," IEEE Transactions on Industrial Informatics, vol. 10, no. 1, pp. 185-196, 2013. https://doi.org/10.1109/TII.2013.2253112

Jānis Pekša is a Research Assistant of the Faculty of Computer Science and Information Technology at Riga Technical University (Latvia). He received his M.Sc. degree in Information Technology from Riga Technical University. Currently he is a Doctoral student at the Information Technology Institute of Riga Technical University. His research interests include modularized implementation of autonomous decision-making algorithms in enterprise resource planning systems.

E-mail: Janis.Peksa@rtu.lv 